\title{
Ectopia lentis et pupillae: relato de caso
}

\author{
Ectopia lentis et pupillae: case report
}

\author{
Cristiane Santos Bernardes ${ }^{1}$ \\ Lívio Viana de Oliveira Leite ${ }^{2}$ \\ Flávia Augusta Attié de Castro ${ }^{3}$
}

Trabalho realizado no Departamento de Oftalmologia da Universidade São Paulo - Setor de Cristalino do Hospital das Clínicas da Faculdade de Medicina de Ribeirão Preto. Ribeirão Preto (SP), Brasil.

${ }^{1}$ Mestre em Oftalmologia pela Faculdade de Medicina de Ribeirão Preto da Universidade de São Paulo (USP). Ribeirão Preto (SP).

${ }^{2}$ Doutor em Oftalmologia pela Faculdade de Medicina de Ribeirão Preto da USP. Ribeirão Preto (SP).

${ }^{3}$ Médica Residente em Oftalmologia da Faculdade de Medicina de Ribeirão Preto da USP. Ribeirão Preto (SP).

Endereço para correspondência: Cristiane S. Bernardes - Rua do Franco, 68 Bloco B apto 14 Campo Grande (MS) CEP 79051-400

E-mail: csantosbernardes@hotmail.com

Recebido para publicação em 22.11.2004

Versão revisada recebida em 04.07.2005

Aprovação em 10.10.2005

\section{RESUMO}

O objetivo do trabalho é relatar um caso de ectopia lentis et pupillae, chamando a atenção para as complicações oculares secundárias e seus diagnósticos diferenciais. Paciente de 27 anos com queixa de baixa acuidade visual. Não possuía características sindrômicas e os exames complementares realizados foram normais. No exame oftalmológico; acuidade visual de conta dedos a 2 metros em olho direito e conta dedos a 1 metro em olho esquerdo. Exotropia alternante de 25 dioptrias. Diâmetros corneanos de $10 \mathrm{~mm}$ e $9 \mathrm{~mm}$ nos meridianos horizontais e verticais, respectivamente. Ceratometria de 39,00 a $178^{\circ}$ x 43,87 a $88^{\circ}$ no olho direito 37,64 a $22^{\circ}$ x 42,75 a $112^{\circ}$ no olho esquerdo. Na biomicroscopia, ambos os olhos apresentavam atrofia iriana, criptas ausentes e transiluminação de íris negativa. Pupila direita com forma regular e tópica, pupila esquerda também de formato regular, mas com deslocamento temporal inferior. Cristalinos opacificados, com tamanhos diminuídos e deslocados nasalmente. Pressão intra-ocular normal. Na ultra-sonografia, comprimento axial de $26 \mathrm{~mm}$ e $30 \mathrm{~mm}$ com diâmetro sagital do cristalino de 5,4 $\mathrm{mm}$ e 4,5 $\mathrm{mm}$ no olho direito e esquerdo, respectivamente. Os diagnósticos diferenciais incluem anormalidades puramente oculares, síndromes e distúrbios metabólicos. O diagnóstico dessa síndrome é importante para avaliação do risco, prognóstico e tratamento, além de ser fundamental seu conhecimento na diferenciação de outras síndromes nas quais há comprometimento sistêmico. A diminuição da acuidade visual é causada, geralmente, por desenvolvimento de grave miopia, catarata, astigmatismo corneano, descolamento de retina e glaucoma sendo justificável acompanhamento oftalmológico regular desses pacientes.

Descritores: Ectopia do cristalino; Anormalidades do olho; Diagnóstico diferencial; Glaucoma; Íris/anormalidades; Relatos de casos [tipo de publicação]

\section{INTRODUÇÃO}

O deslocamento do cristalino é uma desordem conhecida pela comunidade oftalmológica por mais de dois séculos. A sua classificação atual diferencia o deslocamento primário (congênito), secundário e traumático da lente ${ }^{(1)}$. O termo ectopia lentis em particular se refere ao primeiro grupo, ou seja, o deslocamento congênito ou hereditário da lente.

O escopo clínico e genético dessa manifestação tem sido amplamente expandido devido ao reconhecimento de que a ectopia lentis é uma característica comum de várias doenças oculares e sistêmicas na qual avaliação médica completa é essencial ${ }^{(2-3)}$.

Enquanto a síndrome ectopia pupillae é uma anormalidade adquirida, usualmente associada a sérias e freqüentes doenças progressivas do olho, tais como: atrofia essencial da íris, síndrome de Chandler, síndrome de Rieger e inflamação intra-ocular. 
A corectopia congênita é uma entidade não progressiva, rara e poucos oftalmologistas estão familiarizados com esta síndrome na qual a manifestação clínica principal é a associação de deslocamento da pupila e do cristalino. Esta patologia é denominada síndrome ectopia lentis et pupillae e manifesta-se através de transmissão autossômica recessiva com expressão variada. É caracterizada por anormalidades oculares, mas sem manifestações sistêmicas ${ }^{(4-12)}$. Trata-se de uma entidade clínica onde o seu conhecimento permanece ainda insuficiente.

O objetivo deste trabalho é descrever o caso de um paciente com ectopia lentis e pupillae dando ênfase às complicações oculares secundárias à síndrome, e introduzir os diagnósticos diferenciais.

\section{RELATO DE CASO}

MPRF, sexo masculino, 27 anos de idade, natural de Timonha $(\mathrm{CE})$ e procedente de Ribeirão Preto. Seus pais, não consangüíneos, tiveram quatro filhos, mas apenas o segundo apresentou alterações oculares. Relatou possuir primos paternos de primeiro grau também com problemas oftalmológicos, porém desconhecia detalhes específicos. Referiu baixa de visão, bilateral, desde a infância e aos 17 anos de idade iniciou uso de óculos com grau elevado, mas afirmou que sua visão permanecera deficiente mesmo após a correção óptica.

Foram solicitados exames complementares para investigar a possibilidade de comprometimento sistêmico (hemograma, bioquímica geral, proteinograma, sedimento urinário, homocisteína no sangue, aminoácidos no sangue, eletrocardiograma, Rx de tórax e extremidades), mas os resultados não indicaram anormalidade. A estatura do paciente era de $166 \mathrm{~cm}$ e o mesmo não possuía características sindrômicas.

No exame oftalmológico, o paciente apresentava acuidade visual de conta dedos a 2 metros em olho direito e conta dedos a 1 metro em olho esquerdo sem melhora da acuidade visual com correção. Exotropia alternante de 25 dioptrias. Diâmetros corneanos de $10 \mathrm{~mm}$ e $9 \mathrm{~mm}$ nos meridianos horizontais e verticais, respectivamente. Ceratometria de 39,00 a $178^{\circ}$ x 43,87 a $88^{\circ}$ no olho direito e 37,62 a $22^{\circ}$ x 42,75 a $112^{\circ}$ no olho esquerdo.

$\mathrm{Na}$ biomicroscopia, ambos os olhos apresentavam atrofia iriana, criptas ausentes, transiluminação de íris negativa e iridodonese. Pupila direita com forma regular e tópica (Figura 1), pupila esquerda também de formato regular, mas deslocada temporal inferior (Figura 2). Cristalinos opacificados, de tamanhos diminuídos, deslocados súpero-nasalmente (Figura 3).

Pressão intra-ocular de $14 \mathrm{mmHg}$ em ambos os olhos, na gonioscopia evidenciou-se, bilateralmente, ângulo aberto, amplo $360^{\circ}$ e processos irianos proeminentes.

O exame de fundo de olho foi dificultado pela opacidade de meios e pela pouca dilatação pupilar, porém ${ }_{2} \mathrm{o}$ pólo posterior não apresentava alterações. A ultra-sonografia revelou comprimento axial de $26 \mathrm{~mm}$ e cristalino com 5,4 $\mathrm{mm}$ de diâmetro sagital no olho direito e comprimento axial de $30 \mathrm{~mm}$ e cristalino com 4,5 mm de diâmetro sagital no olho esquerdo.

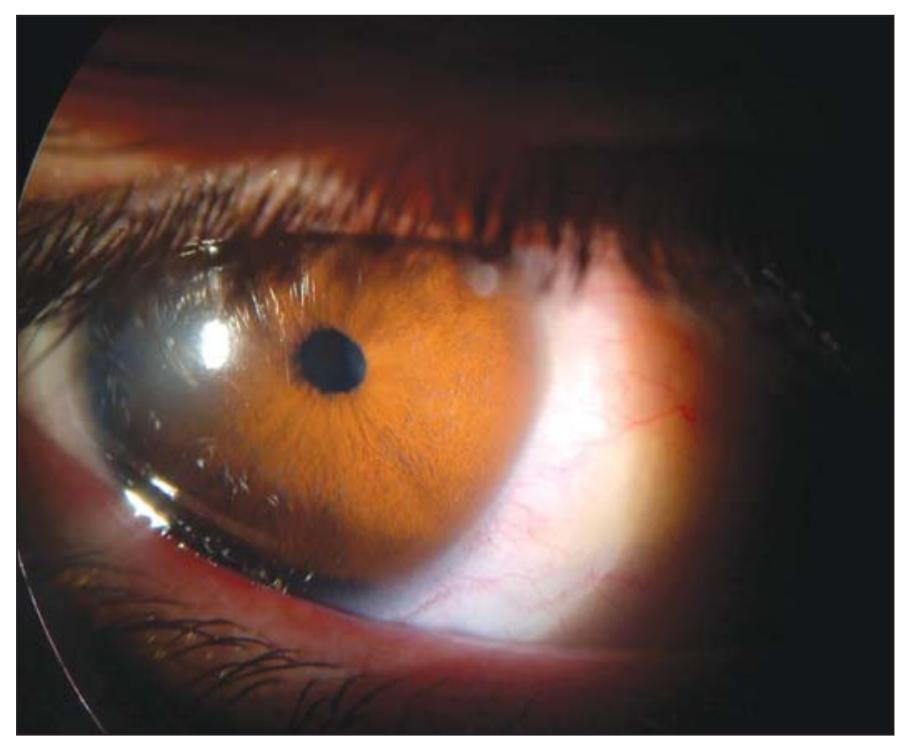

Figura 1 - Olho direito com pupila centrada, forma regular e ausência de criptas irianas

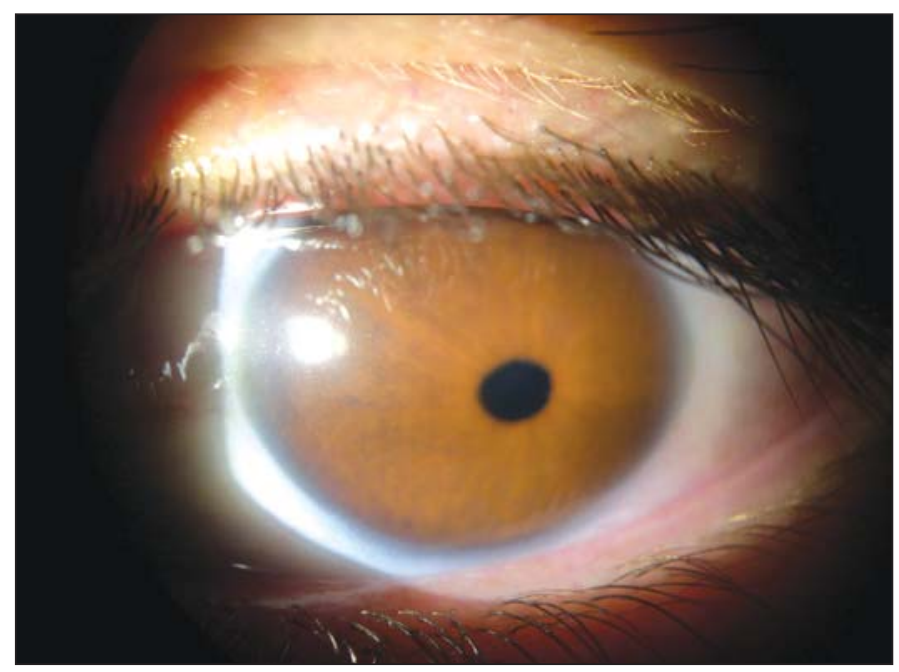

Figura 2 - Olho esquerdo com deslocamento pupilar temporal inferior

O paciente está sendo acompanhado no ambulatório de cristalino e sua programação cirúrgica inicial prevê a realização de cirurgia de catarata por facoemulsificação com o auxílio de anel expansor intracapsular.

O diagnóstico diferencial de ectopia lentis e pupillae incluem anormalidades puramente oculares, síndromes e desordens metabólicas (Tabela 1$)^{(4)}$.

\section{DISCUSSÃO}

Deslocamento do cristalino é um importante diagnóstico devido à possibilidade de estar associado a síndromes ou a doenças sistêmicas ${ }^{(2-3)}$. Subluxação do cristalino pode ocorrer em qualquer direção e uma posição particular é apenas sugestiva de uma condição específica. 


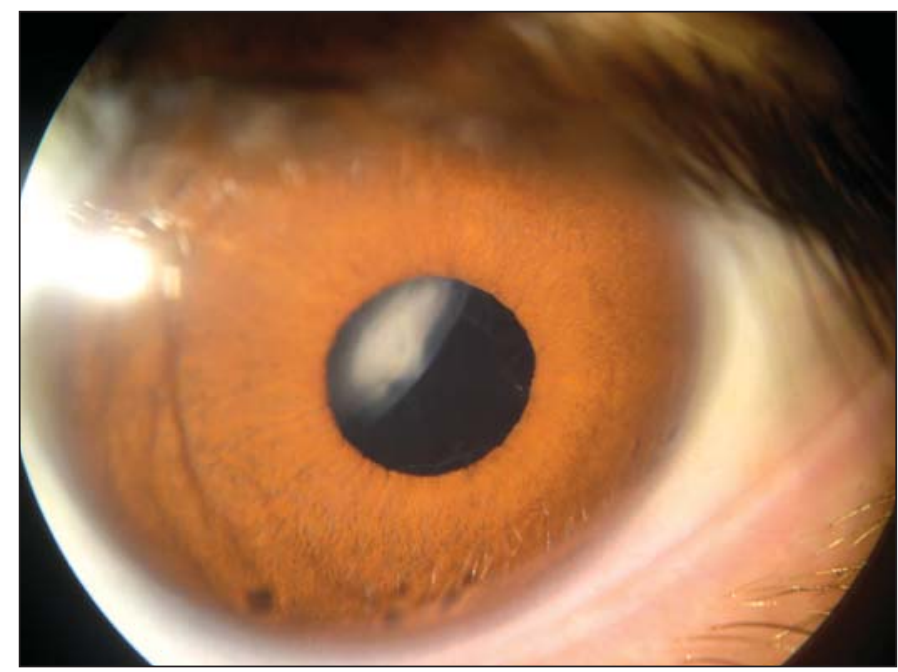

Figura 3 - Olho esquerdo em midríase máxima com cristalino opacificado e deslocado nasal superior

Há três tipos básicos de ectopia lentis hereditária: (1) ectopia lentis associada com malformações generalizadas ou doenças sistêmicas; (2) ectopia lentis sem associação a problemas sistêmicos e (3) ectopia lentis et pupillae sem associação a problemas sistêmicos ${ }^{(5)}$.

A pupila humana normal está deslocada $0,5 \mathrm{~mm}$ inferior e nasalmente. $\mathrm{O}$ deslocamento pupilar patológico está freqüentemente associado com doença ocular progressiva, mas também pode ser congênita não progressiva. Nesta situação, deslocamento do cristalino também é encontrado com freqüência, mas pode ser difícil sua detecção se o exame não for realizado sob midríase. Outros achados como iridodonese, piora progressiva da acuidade visual e mudanças abruptas na refração podem ser importantes para a caracterização da síndrome ectopia lentis et pupillae ${ }^{(6)}$.

Ectopia lentis et pupillae foi descrita no início do século 20, em publicação alemã, e foi amplamente demonstrado tratar-se de doença rara, com herança autossômica recessiva, com múltiplas ocorrências de membros da mesma família não afetados e associação a casamentos consangüíneos ${ }^{(4)}$.

A patogênese dessa síndrome não é completamente compreendida. Originalmente, acreditava-se tratar de um tipo de anormalidade iriana. Mais recentemente, anormalidades no desenvolvimento neuroectodermal tem sido postulado ${ }^{(7)}$.

Ambos os olhos são usualmente afetados e podem ser imagem em espelho um do outro, embora a condição possa ser unilateral. A acuidade visual é dependente do formato pupilar e da ectopia do cristalino. As pupilas são muitas vezes discóricas, em forma de fenda, elipse ou oval, porém em $40 \%$ dos pacientes elas são normais, ocorre limitação ou ausência de dilatação em $60 \%$ dos casos devido atrofia e transiluminação positiva da íris ocorre também em $60 \%$ dos $\operatorname{casos}^{(8)}$. Nosso paciente não apresentava alteração na forma pupilar nem transiluminação positiva da íris, mas foi verificada limitação para dilatação pupilar em ambos os olhos.

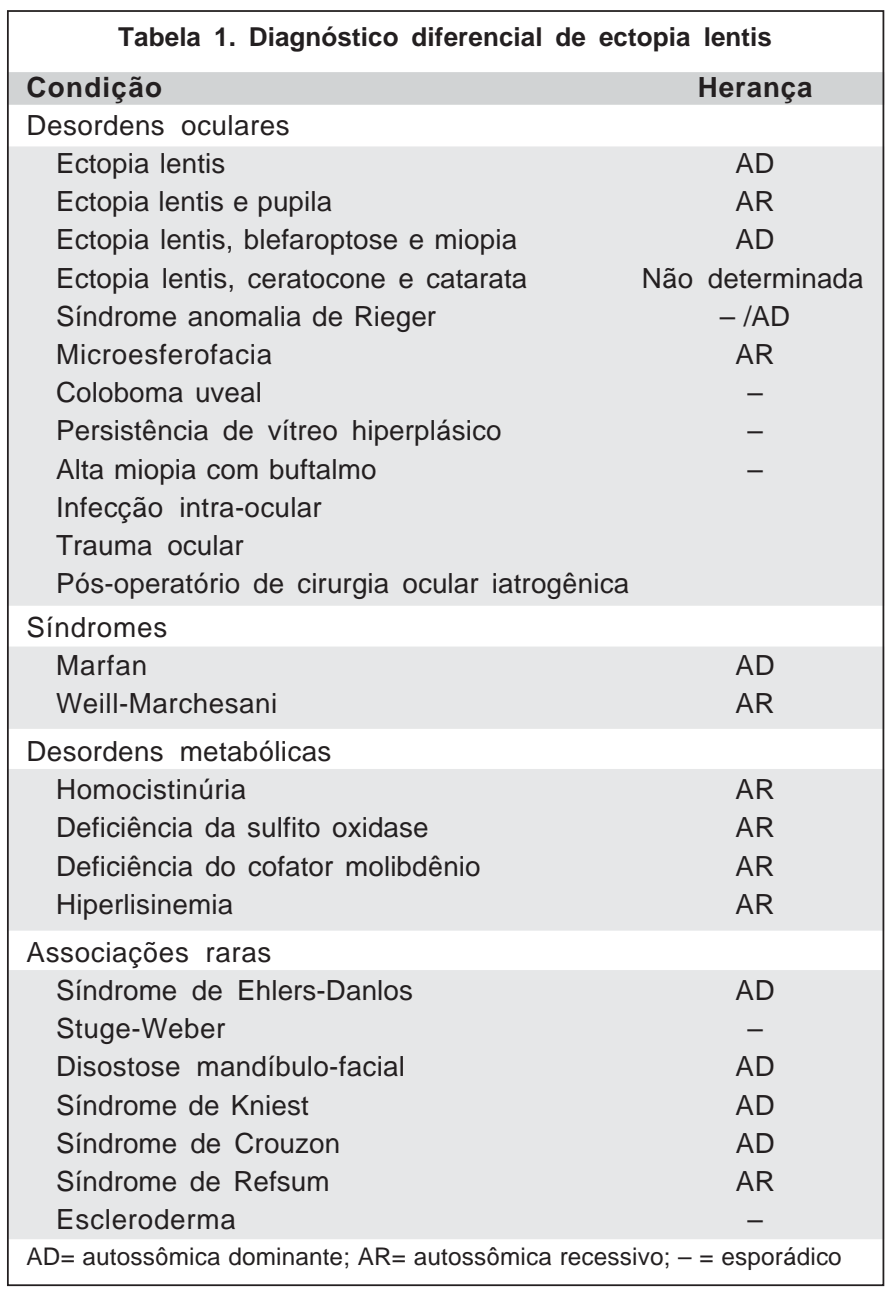

Townes descreveu que nessa síndrome os processos irianos são proeminentes e a pupila pode ser deslocada em direção oposta à ectopia do cristalino ${ }^{(9)}$. Outros relatos indicam que o cristalino pode estar deslocado em qualquer direção ${ }^{(10)}$. Podem-se ter casos onde há apenas ectopia da lente, bilateralmente, e há estudos onde se tem deslocamento do cristalino em um olho e deslocamento do cristalino e pupila no olho adelfo, conforme descrito por Fecht* em $1927^{(10)}$.

No exame gonioscópico do paciente estudado, os processos irianos eram proeminentes, os cristalinos estavam deslocados ambos para a mesma posição (súpero-nasal) e apenas a pupila do olho esquerdo deslocada em sentido contrário ao do cristalino.

Outros achados no exame oftalmológico deste paciente foram: miopia axial, astigmatismo corneano, opacidade de cristalino e estrabismo. Alterações oculares concordantes com dados encontrados na literatura ${ }^{(1,5-6,8-9,9,11)}$.

Uma observação importante deste estudo é que não encontramos a associação, freqüente, megalocórnea - ectopia lentis et

* Fecht W. Ueber familiäre linsenluxation. Z Augenheilkd. 1927;62:162-8. apud ${ }^{(10)}$ 
pupillae $e^{(6-7,11,13)}$. Contrariando esta expectativa, nosso paciente apresenta microcórnea. Não foi encontrado nenhum relato desta inusitada associação durante nossa busca pela literatura.

Outras alterações que podem estar associadas a esta síndrome incluem: embriotoxon posterior, persistência de membranas pupilares, adesão irido-hialoidiana e uveíte anterior ${ }^{(9-12)}$. Deslocamento do cristalino para câmara anterior ou posterior e estrabismo também podem ocorrer ${ }^{(6)}$.

No relato de Goldberg foram estudados 16 pacientes com ectopia lentis et pupillae ${ }^{(11)}$ e foram encontrados: deslocamento de pupila em $60 \%$ dos olhos, alta miopia e disco óptico inclinado ocorreu em mais de $50 \%$ dos pacientes, diâmetro corneano aumentado e astigmatismo corneano foram encontrados em mais de um terço dos olhos afetados, catarata também ocorreu em aproximadamente um terço dos olhos e descolamento de retina em $9 \%$. Glaucoma não foi relatado como sendo comum neste estudo.

Com relação à associação dessa síndrome ao glaucoma há discordância entre autores, enquanto Townes ${ }^{(9)}$, Cruysberg, Pinckers ${ }^{(12)}$ e Byles et al. ${ }^{(7)}$, encontraram esta associação em seus respectivos estudos, outros autores ${ }^{(4,6,11)}$ concordam que não se trata de uma associação comum. Nosso paciente não apresentava aumento da pressão intra-ocular nem alterações de disco óptico sugestivas de glaucoma.

O correto diagnóstico de ectopia lentis et pupillae é importante para avaliação do risco, prognóstico e tratamento. Além de ser fundamental seu conhecimento para realização de diagnóstico diferencial com outras síndromes onde há comprometimento sistêmico.

A diminuição da acuidade visual ao longo da vida é causada mais freqüentemente por desenvolvimento de severa miopia, catarata, astigmatismo corneano e descolamento de retina $^{(4)}$ sendo justificável portanto, acompanhamento oftalmológico regular desses pacientes.

\section{ABSTRACT}

The main purpose of this report is to describe a case of ectopia lentis et pupillae syndrome, highlighting the secondary ocular complications and their differential diagnoses. A 27-year-old man presented with complaint of low visual acuity. No evidence of the syndrome was found at presentation. The results of supplementary tests were normal. On ophthalmologic examination, visual acuity was finger count at 2 meters in the right eye and finger count at 1 meter in the left eye. Exotropia of 25 dioptrics. Corneal diameters of $10 \mathrm{~mm}$ and $9 \mathrm{~mm}$ in the horizontal and vertical meridians, respectively. Keratometry readings of 39.00 at $178 \times 43.87$ at 88 for the right eye and 37.64 at $22 \times 42.75$ at 122 for the left eye. The biomicroscopic examination revealed iris atrophy in both eyes, absence of crypt and absent iris transillumination. Centralized right pupil with regular form and left pupil with inferior-temporal dislocation. Opaque lenses with reduced sizes and superior-nasal dislocation. Normal intraocular pressure. Axial length of $26 \mathrm{~mm}$ and $30 \mathrm{~mm}$ and crystalline axial diameter of 5.4 and $4.5 \mathrm{~mm}$ in right and left eyes, respectively, as revealed by ultrasonography. The differential diagnosis encompasses exclusively ocular abnormalities, syndromes and metabolic disorders. Diagnosis of the ectopia lentis et pupillae is fundamental not only to evaluate the risk, prognosis and treatment, but also to assist in differentiating other syndromes with systemic impairment. A visual acuity reduction is normally caused by severe myopia development, cataract, corneal astigmatism, retinal detachment and glaucoma, justifying regular ophthalmologic support for these patients.

Keywords: Ectopia lentis; Eye abnormalities; Diagnosis, differential; Glaucoma; Iris/abnormalities

\section{REFERÊNCIAS}

1. Fuchs, J, Rosenberg, T. Congenital ectopia lentis. A Danish national survey. Acta Ophthalmol Scand. 1998:76(1):20-6.

2. Cross HE, Jensen AD. Ocular manifestations in the Marfan syndrome and homocystinuria. Am J Ophthalmol. 1973;75(3):405-20.

3. Pagon RA. Ophthalmic manifestations of metabolic disorders. Proc Greenwood Genet Center. 1989;8:97-107.

4. Colley A, Lloyd IC, Ridgway A, Donnai D. Ectopia lentis et pupillae: the genetic aspects and differential diagnosis. J Med Genet. 1991;28(11):791-4.

5. Jensen A. Heritable ectopia lentis. In: Goldberg MF, editor. Genetic and metabolic eye disease. Boston: Little, Brown;1974. p.325.

6. Cross HE. Ectopia lentis et pupillae. Am J Ophthalmol. 1979;88(3 Pt 1):381-4.

7. Byles DB, Nischal KK, Cheng H. Ectopia lentis et pupillae. A hypothesis revisited. Ophthalmology. 1998;105(7):1331-6.

8. Pagon Roberta A, Spaeth George L. Congenital malformations of the eye. In: Tasman W, Jaeger EA, editors. Duane's Foundations of Clinical Ophthalmology. Philadelphia: Lippincott-Raven; 1995. v. 1. p.29.

9. Townes PL. Ectopia lentis et pupillae. Arch Ophthalmol. 1976;94(7):1126-8.

10. Nelson LB, Maumenee IH. Ectopia lentis. Surv Ophthalmol. 1982;27(3):143-60.

11. Goldberg MF. Clinical manifestations of ectopia lentis et pupillae in 16 patients. Ophthalmology. 1988;95(8):1080-7.

12. Cruysberg JRM, Pinckers A. Ectopia lentis et pupillae syndrome in three generations. Br J Ophthalmol. 1995;79(2):135-8.

13. Luebbers AJ, Goldberg MF, Herbst R, Hattenhauer J, Maumenee AE. Iris transillumination and variable expression in ectopia lentis et pupillae. Am J Ophthalmol. 1977;83(5):647-55. 